\title{
Contour and surface integrals in potential scattering
}

\author{
Giampiero Esposito, INFN Sezione di Napoli, \\ Complesso Universitario di Monte S. Angelo, \\ Via Cintia, Edificio 6, 80126 Napoli, Italy
}

5 February 2020

\begin{abstract}
When the Schrödinger equation for stationary states is studied for a system described by a central potential in $n$-dimensional Euclidean space, the radial part of stationary states is an even function of a parameter $\lambda$ which is a linear combination of angular momentum quantum number $l$ and dimension $n$, i.e., $\lambda=l+\frac{(n-2)}{2}$. Thus, without setting a priori $n=3$, complex values of $\lambda$ can be achieved, in particular, by keeping $l$ real and complexifying $n$. For suitable values of such an auxiliary complexified dimension, it is therefore possible to obtain results on scattering amplitude and phase shift that are completely equivalent to the results obtained in the sixties for Yukawian potentials in $\mathbf{R}^{3}$. Moreover, if both $l$ and $n$ are complexified, the possibility arises of recovering the partial wave amplitude from residues of a function of two complex variables. Thus, the complex angular momentum formalism can be imbedded into a broader framework, where a correspondence exists between the scattering amplitude and a skew curve in $\mathbf{R}^{3}$.
\end{abstract}

\section{Introduction}

The theory of potential scattering has been always of fundamental importance in quantum mechanics, since it provides the appropriate tool for the investigation of matter at nuclear and subnuclear level. In this paper, we are interested in obtaining a new perspective on potential scattering in ordinary (i.e., nonrelativistic) quantum mechanics. For this purpose, our starting point is the familiar form of the scattering amplitude when the partial-wave expansion is applied in presence of Yukawian central potentials, i.e. [1, 2, 3]

$$
f(k, \cos \theta)=\frac{1}{2 i k} \sum_{l=0}^{\infty}(2 l+1)\left(e^{2 i \delta(l, k)}-1\right) P_{l}(\cos \theta),
$$

where $\theta$ is the angle between initial wave vector $\vec{k}_{\mathrm{i}}$ and final wave vector $\vec{k}_{\mathrm{f}}, \delta(l, k)$ is the phase shift and $P_{l}$ is the standard notation for Legendre polynomials. The work in Refs. 1, 2, 3, had the merit, among the many, of recognizing that all terms in this sum can be obtained by applying the residue theorem to a contour 
integral, where the function to be integrated is

$$
Q_{k}: \lambda \in \mathbf{C} \rightarrow Q_{k}(\lambda)=\frac{1}{2 k}\left(e^{2 i \delta(\lambda, k)}-1\right) \frac{\lambda P_{\lambda-\frac{1}{2}}(-\cos \theta)}{\cos (\pi \lambda)},
$$

and the integration path $\gamma$ encircles all zeros of $\cos (\pi \lambda)$ on the positive half-line $\operatorname{Re}(\lambda)>0$, which are given by $l+\frac{1}{2}, \forall l=0,1, \ldots, \infty$. Indeed, one finds

$$
\begin{aligned}
& \left.\operatorname{Res} Q_{k}(\lambda)\right|_{\lambda=l+\frac{1}{2}}=\lim _{\lambda \rightarrow l+\frac{1}{2}}\left(\lambda-l-\frac{1}{2}\right) Q_{k}(\lambda) \\
= & \frac{(2 l+1)}{4 k}\left(e^{2 i \delta(l, k)}-1\right) P_{l}(-\cos \theta) \lim _{\varepsilon \rightarrow 0} \frac{\varepsilon}{\cos \left(\pi l+\frac{\pi}{2}+\pi \varepsilon\right)} \\
= & \frac{(2 l+1)}{4 k}\left(e^{2 i \delta(l, k)}-1\right) P_{l}(-\cos \theta) \lim _{\varepsilon \rightarrow 0} \frac{\varepsilon}{[-\pi \varepsilon \cos (\pi l)]} \\
= & -\frac{(2 l+1)}{4 \pi k}\left(e^{2 i \delta(l, k)}-1\right) P_{l}(\cos \theta),
\end{aligned}
$$

since $\cos (\pi l)=(-1)^{l}$, and $(-1)^{l} P_{l}(-\cos \theta)=P_{l}(\cos \theta)$. One therefore finds that

$$
\begin{aligned}
& \int_{\gamma} Q_{k}(\lambda) d \lambda=2 \pi i \sum_{l=0}^{\infty}\left[-\frac{(2 l+1)}{4 \pi k}\left(e^{2 i \delta(l, k)}-1\right) P_{l}(\cos \theta)\right] \\
= & f(k, \cos \theta) .
\end{aligned}
$$

In other words, in order to obtain from the residue theorem the countable infinity of contributions to the scattering amplitude, it is not enough to consider the contour integral of a rational function. One needs instead the ratio of a polynomial and a cosine function. The latter will also contribute $(-1)^{l}$ factors that turn $P_{l}(-\cos \theta)$ into $P_{l}(\cos \theta)$, eventually. The integral on the left-hand side of Eq. (1.4) can be rotated with profit to the imaginary axis $\operatorname{Im}(\lambda)$, going from $-i \infty$ to $+i \infty$. The resulting integral is therefore a Watson transform [3, 4, 5, 6].

On the other hand, it is well known that central potentials can be studied with profit in $n$-dimensional Euclidean space $\mathbf{R}^{n}$ [7. In section 2, relying upon our previous work 8 , we consider the role of the $\lambda$-parameter in the Schödinger equation for stationary states. Sections 3 and 4 are devoted to the cases real $l$ and complex $n$, complex $l$ and complex $n$, respectively. Concluding remarks are made in section 5 .

\section{The $\lambda$ parameter}

As is shown, for example, in Refs. [7, 8, the Schrödinger equation for stationary states in $n$-dimensional Euclidean space (whose consideration is inspired by the large- $n$ formalism in quantum mechanics) leads to the following equation for the radial part $\psi(r)$ of stationary states:

$$
\left[\frac{d^{2}}{d r^{2}}+\frac{(n-1)}{r} \frac{d}{d r}+\frac{2 m}{\hbar^{2}}(E-U(r))-\frac{l(l+n-2)}{r^{2}}\right] \psi(r)=0 .
$$


This equation must be solved with suitable boundary conditions as $r \rightarrow 0^{+}$and as $r \rightarrow \infty$, and the full stationary state reads eventually as

$$
\psi(r) g\left(\theta_{1}, \ldots, \theta_{n-1}\right),
$$

where $g$ is the generalized spherical harmonic. At this stage, a technique already developed by Liouville 9 for studying linear differential equations with variable coefficients suggests setting

$$
\psi(r)=r^{\beta} \varphi(r),
$$

where $\beta=\beta(n)$ should be chosen in such a way that the resulting differential equation for the unknown function $\varphi$ has vanishing coefficient of $\frac{d \varphi}{d r}$. One then finds that the desired $\beta(n)$ should solve the algebraic equation of first degree

$$
2 \beta+(n-1)=0 \Longrightarrow \beta=-\frac{(n-1)}{2} .
$$

On defining

$$
k^{2} \equiv \frac{2 m E}{\hbar^{2}}, V(r) \equiv \frac{2 m}{\hbar^{2}} U(r),
$$

Eqs. (2.1)-(2.4) lead to the following second-order equation for $\varphi(r)$ :

$$
\left[\frac{d^{2}}{d r^{2}}-\left(l(l+n-2)+\frac{1}{4}\left(n^{2}-4 n+3\right)\right) \frac{1}{r^{2}}+k^{2}-V(r)\right] \varphi(r)=0 .
$$

Interestingly, by exploiting the identity 8

$$
l(l+n-2)=\left(l+\frac{(n-2)}{2}\right)^{2}-\frac{1}{4}(n-2)^{2},
$$

one finds that

$$
l(l+n-2)+\frac{1}{4}\left(n^{2}-4 n+3\right)=\left(l+\frac{(n-2)}{2}\right)^{2}-\frac{1}{4},
$$

and hence Eq. (2.5) takes eventually the form

$$
\left[\frac{d^{2}}{d r^{2}}+k^{2}-\frac{\left(\lambda^{2}-\frac{1}{4}\right)}{r^{2}}-V(r)\right] \varphi(r)=0,
$$

having defined

$$
\lambda=\lambda(l, n) \equiv l+\frac{(n-2)}{2} .
$$

With the modern language of quantum mechanics one can say that a unitary operator $U$ has been built that realizes the map [10]

$$
U: L^{2}\left(\mathbf{R}^{+}, r^{n-1} d r\right) \rightarrow L^{2}\left(\mathbf{R}^{+}, d r\right) .
$$

The parameter denoted by $\nu(l)$ in Ref. [10] coincides with our $\lambda$ defined in Eq. (2.9), and we refer the reader to Ref. 10 for many important results obtained with arbitrary integer values of $n$ in Eq. (2.9). 


\section{Real $l$ and complex $n$}

Since the formalism suggests considering complex values of $\lambda$, and Eq. (2.8) bears full resemblance with the equation for $\varphi(r)$ in $\mathbf{R}^{3}[3$, we can in particular achieve complex values of $\lambda$ by keeping $l$ real while allowing for complexified values of $n$. In other words, given a pair $\left(l_{1}, n_{1}\right)$ where $l_{1} \in \mathbf{C}, n_{1}=3$ (this is the case studied in Refs. [1, 2, 3]), and another pair $\left(l_{2}, n_{2}\right)$ where $l_{2} \in \mathbf{R}, n_{2} \in \mathbf{C}$, if we require that

$$
\lambda\left(l_{1}, n_{1}=3\right)=\lambda\left(l_{2}, n_{2}\right),
$$

we find

$$
\begin{gathered}
\operatorname{Re}\left(n_{2}\right)=2 \operatorname{Re}\left(l_{1}\right)+3-2 l_{2}, \\
\operatorname{Im}\left(n_{2}\right)=2 \operatorname{Im}\left(l_{1}\right) .
\end{gathered}
$$

If such conditions are fulfilled, we can import all results obtained in Ref. 3], and hence write that, at large $|\lambda|$ with $\operatorname{Re}(\lambda)=l_{2}+\frac{1}{2} \operatorname{Re}\left(n_{2}\right)-1>0$, along the ray $\arg (\lambda)=\sigma$, one finds

$$
\left|\frac{\lambda P_{\lambda-\frac{1}{2}}(-\cos \theta)}{\cos (\pi \lambda)}\right| \leq \frac{\kappa_{1} \sqrt{|\lambda|}}{\sqrt{|\sin \theta|}} \exp \{-|\operatorname{Re}(\theta) \operatorname{Im}(\lambda)|+\operatorname{Im}(\theta) \operatorname{Re}(\lambda)\} .
$$

Moreover, if the potential $V(r)$ is Yukawian, 1 , then for large enough $|\lambda|$, but $\arg (\lambda) \neq \frac{\pi}{2}$, one finds $[3$

$$
\left|e^{2 i \delta(\lambda, k)}-1\right| \leq \kappa_{2} \exp [-\alpha \operatorname{Re}(\lambda)],
$$

where $\alpha$ is such that $\cosh (\alpha)=1+\frac{m^{2}}{2 k^{2}}$. By virtue of (3.4) and (3.5) one then obtains the important majorization [3]

$$
\left|2 k Q_{k}(\lambda)\right| \leq \kappa \sqrt{|\lambda|} \exp \{-|\operatorname{Re}(\theta) \operatorname{Im}(\lambda)|+\operatorname{Im}(\theta) \operatorname{Re}(\lambda)-\alpha \operatorname{Re}(\lambda)\} .
$$

\section{Complex $l$ and complex $n$}

The definition (2.9) suggests investigating the more general case when both $l$ and $n$ are complex. The resulting framework is non-trivial, because we are then asking whether Eq. (1.1) can be viewed as arising from the residue of a double integral of a function of two complex variables, $l$ and $n$. In other words, the

\footnotetext{
${ }^{1}$ Recall from Ref. 3 that the potential $V(z)$ is Yukawian if one can express it in the form

$$
V(z)=\int_{m}^{\infty} C(\mu) e^{-\mu z} d \mu
$$

where $C(\mu)$ is a distribution. Other relevant cases occur when $C(\mu)$ is a function of bounded variation, for which the potential reads as

$$
V(z)=\int_{m}^{\infty} \frac{e^{-\mu z}}{z} d C_{\mu}
$$

or when $C$ is an absolutely continuous function with first derivative $C^{\prime}(\mu)=\sigma(\mu)$, for which $V(z)$ takes the form

$$
V(z)=\int_{m}^{\infty} \frac{e^{-\mu z}}{z} \sigma(\mu) d \mu .
$$

The Yukawa potential is recovered from the first formula when $C(\mu)$ is just a constant.
} 
Regge formslism in the form developed in the sixties and until now is part of a broader framework, and it is our task to define properly such a framework and then investigate its potentialities.

\subsection{Double integrals in $\mathrm{C}^{2}$}

For this purpose, we begin by describing some basic properties of complex integration of expressions like

$$
I=\iint_{\sigma} F(\xi, \eta) d \xi d \eta
$$

following the pioneering work of Poincaré [1], who was the undisputed founder of this research line (Leray provided the modern rigorous theory in Ref. [12, for which we refer also to Ref. [13]).

A double integral must be evaluated on a surface, denoted by $\sigma$ in (4.1). If the function $F$ can be expressed by the ratio $\frac{p(\xi, \eta)}{q(\xi, \eta)}$, it becomes of course infinite when the denominator $q(\xi, \eta)$ vanishes. Such a condition leads in turn to the pair of equations (we set $\xi=x+i y, \eta=z+i t$ )

$$
\begin{aligned}
& \operatorname{Re} q(\xi, \eta)=q_{1}(x, y, z, t)=0, \\
& \operatorname{Im} q(\xi, \eta)=q_{2}(x, y, z, t)=0 .
\end{aligned}
$$

Equations (4.2) and (4.3) are the defining equations of the singular surface $\Sigma_{s}$, and we require that the integration surface $\sigma$ has empty intersection with $\Sigma_{s}$.

Let $(\lambda, \mu, \nu)$ be the coordinates of a point in $\mathbf{R}^{3}$, and let us consider an algebraic surface $S$, or portion of an algebraic surface, upon which the point $(\lambda, \mu, \nu)$ lies. On writing

$$
x=\varphi_{1}(\lambda, \mu, \nu), y=\varphi_{2}(\lambda, \mu, \nu), z=\varphi_{3}(\lambda, \mu, \nu), t=\varphi_{4}(\lambda, \mu, \nu),
$$

we may assume that $\varphi_{1}, \varphi_{2}, \varphi_{3}, \varphi_{4}$ are rational functions of $\lambda, \mu, \nu$ whose denominator never vanishes. By construction, when the point $(\lambda, \mu, \nu)$ describes in $\mathbf{R}^{3}$ the surface (or portion of surface) $S$, the point $(x, y, z, t)$ describes a surface (or portion of surface) $S^{\prime}$. The resulting surface $S^{\prime}$ is defined by the surface $S$ and by the four fundamental functions $\varphi_{1}, \varphi_{2}, \varphi_{3}$ and $\varphi_{4}$. We can also work under weaker assumptions, and hence assume that $\varphi_{1}, \varphi_{2}, \varphi_{3}$ and $\varphi_{4}$ in (4.4) are just single-valued analytic functions of $\lambda, \mu, \nu$ at least in a certain subset of $\mathbf{R}^{3}$, in such a way that the surface $\sigma$ that represents the integration surface is entirely contained within such a subset.

In order to ascribe a meaning to a formula like (4.1), we must also define the concepts of inward- or outward-pointing. For this purpose, we can imagine an observer $\mathcal{O}$ standing on the surface $S$ with his/her head directed towards the interior of the surface. The position of such an observer defines the sense of integration. Following Poincaré [1], the sense of integration is positive (resp. negative) if the observer's head is directed towards the exterior (resp. interior). If the surface $S$ is not closed, we can nevertheless agree that one of the sides is the exterior, while the other side is then said to represent the interior. On setting $F=P+i Q$, the formal manipulation of (4.1) would yield

$$
I=\iint(P+i Q)(d x+i d y)(d z+i d t)
$$




$$
\begin{aligned}
& =\iint[(P+i Q) d x d z+(i P-Q) d x d t \\
& +\quad(i P-Q) d y d z-(P+i Q) d y d t] .
\end{aligned}
$$

If we now assume that two auxiliary variables $u, v$ exist such that, at all points of the surface $S$, the three coordinates $\lambda, \mu, \nu$ are holomorphic functions of $u$ and $v$, the desired integral (4.5) becomes the ordinary double integral

$$
\begin{array}{r}
\iint\left[(P+i Q) \frac{\partial(x, z)}{\partial(u, v)}+(i P-Q) \frac{\partial(x, t)}{\partial(u, v)}\right. \\
\left.+\quad(i P-Q) \frac{\partial(y, z)}{\partial(u, v)}-(P+i Q) \frac{\partial(y, t)}{\partial(u, v)}\right] d u d v,
\end{array}
$$

extended over all values of $u$ and $v$, and which correspond to the different points of the surface $S$ described by $(\lambda, \mu, \nu)$ as they vary. More precisely, we may have to decompose the surface $S$ into a number of surfaces of smaller measure, within each of which two variables $u, v$ exist such that $\lambda, \mu, \nu$ are holomorphic functions of $u$ and $v$.

Note that the integral (4.6) changes sign when a permutation of $u$ and $v$ is performed. In order to fix a convention, let us imagine that $u, v$ represent the coordinates of a point in the plane. Let us also imagine that the point $(\lambda, \mu, \nu)$ describes on the surface $S$ a very small closed contour $C$ about the feet of the observer $\mathcal{O}$, and that this observer sees this point as describing anticlockwise the contour $C$. The corresponding point $(u, v)$ describes in its plane another closed contour $C^{\prime}$. The contour $C^{\prime}$ must be traveled anticlockwise as it occurs for the contour $C$ on $S$, assuming that the $u>0$ and $v>0$ axes are arranged as the $x>0$ and $y>0$ axes. The integrability conditions for (4.6) read

$$
\begin{aligned}
\frac{\partial}{\partial x}(i P-Q)-\frac{\partial}{\partial y}(P+i Q) & =0, \\
-\frac{\partial}{\partial x}(P+i Q)-\frac{\partial}{\partial y}(i P-Q) & =0, \\
\frac{\partial}{\partial t}(P+i Q)-\frac{\partial}{\partial z}(i P-Q) & =0, \\
\frac{\partial}{\partial t}(i P-Q)+\frac{\partial}{\partial z}(P+i Q) & =0 .
\end{aligned}
$$

Let us consider the portions of surfaces $S$ and $S^{\prime}$ bounded by the same contour $C$, assuming that both portions lie on the three-space with coordinates $\lambda, \mu, \nu$. Let the two integration surfaces be defined by $S$ and $S^{\prime}$, respectively, but having the same fundamental equations (4.4). If the surface $S$ can, by continuous deformation, coincide with $S^{\prime}$, and if during such a deformation it never happens that $F=P+i Q$ becomes infinite or discontinuous at a point of the integration surface, the integral evaluated on $S$ equals the integral evaluated on $S^{\prime}$.

On the other hand, the singular surfaces where $|F|=\infty$ or $F$ is discontinuous, are described by a pair of equations (cf. (4.2) and (4.3))

$$
\phi_{1}(x, y, z, t)=0, \phi_{2}(x, y, z, t)=0 .
$$


If we here re-express $x, y, z, t$ by means of Eqs. (4.4), the two equations for singular surfaces reduce to the relations

$$
f_{1}(\lambda, \mu, \nu)=0, f_{2}(\lambda, \mu, \nu)=0
$$

among $\lambda, \mu$ and $\nu$. Such equations describe skew curves in $\mathbf{R}^{3}$ with coordinates $\lambda, \mu, \nu$, and such curves are said to be the singular curves, because they are the set of all points $(\lambda, \mu, \nu)$ of $\mathbf{R}^{3}$ where $|F|=\infty$ or $F$ becomes discontinuous. To sum up, one of the following cases may occur:

(i) The two portions of surface $S$ and $S^{\prime}$ being bounded by the same contour $C$, they divide the 3 -space with coordinates $\lambda, \mu, \nu$ into two regions, one internal and the other external. If in the internal region there is no point belonging to singular curves, the integral (4.1) evaluated on $S$ equals the integral evaluated on $S^{\prime}$.

(ii) If the surface $S$ is closed, it divides the 3 -space with coordinates $\lambda, \mu, \nu$ into two regions; if within $S$ there is no point lying on singular curves, the integral evaluated on $S$ vanishes.

(iii) If the surface $S^{\prime}$ is closed as $S$ and entirely contained within $S$, and if in the space between $S$ and $S^{\prime}$ there is no point lying on singular curves, the integral evaluated on $S$ equals the integral evaluated on $S^{\prime}$.

(iv) If two surfaces $S$ and $S^{\prime}$, both closed and belonging to 3 -space with coordinates $\lambda, \mu, \nu$, contain inside them the same singular curves and the same portions of singular curves, the integral (4.1) evaluated on $S$ equals the integral evaluated on $S^{\prime}$.

In the simplest possible terms, a double integral taken along a closed surface $S$ can only depend on the singular curves contained within such a surface 11 . We therefore consider the double integral (4.1) taken over a closed surface $S$, the observer $\mathcal{O}$ being outward-pointing. Inspired by the previous sections, we assume that

$$
F(\xi, \eta)=\frac{P(\xi, \eta)}{Q(\xi, \eta)},
$$

where $P$ is a polynomial while $Q$ has zeros corresponding to the singular curves, i.e.,

$$
Q\left[\varphi_{1}(\lambda, \mu, \nu)+i \varphi_{2}(\lambda, \mu, \nu), \varphi_{3}(\lambda, \mu, \nu)+i \varphi_{4}(\lambda, \mu, \nu)\right]=0 .
$$

Unlike the theory developed by Poincare2, our $Q(\xi, \eta)$ is not a polynomial, but remains responsible for the meromorphic nature of $F(\xi, \eta)$.

Let us assume that the surface $S$ contains two singular curves, e.g., $C$ and $C^{\prime}$. We can always build in $\mathbf{R}^{3}$ with points $(\lambda, \mu, \nu)$ two closed surfaces $\Sigma$ and $\Sigma^{\prime}$, both contained within $S$, and such that $\Sigma$ (resp. $\Sigma^{\prime}$ ) contains only $C$ (resp. $C^{\prime}$ ). The integral (4.1), when taken over $S$, is then the sum of the integral taken over $\Sigma$ and the integral taken over $\Sigma^{\prime}$, the observer $\mathcal{O}$ being always directed outwards. Eventually, we revert to the case where the integration surface $S$ contains just one singular curve $C$.

\footnotetext{
${ }^{2}$ The assumption of Poincaré is not however too restrictive, since a theorem holds, according to which a function that is meromorphic over the whole of $\mathbf{C}^{2}$, including the point at infinity, is a rational function [14].
} 
It is not necessary for the various surfaces $S$ to belong to the same space with points $(\lambda, \mu, \nu)$. Let us therefore consider a particular space with points denoted instead by $\left(\lambda^{\prime}, \mu^{\prime}, \nu^{\prime}\right)$ that nevertheless contains the singular curve $C$ and, within such a space, a closed surface $\Sigma$ enclosing such a curve. The idea is to choose this surface in such a way that integration of $F$ over $S$ equals, up to a sign, the integration of $F$ over $\Sigma$.

Indeed, since the singular curve $C$ is closed, its equations can be always written in the form

$$
x=\psi_{1}(\omega), y=\psi_{2}(\omega), z=\psi_{3}(\omega), t=\psi_{4}(\omega),
$$

the $\psi$ 's being periodic functions of the parameter $\omega$. Following Poincaré [1], their period is here set to $2 \pi$. As a next step, we introduce two auxiliary parameters $\rho$ and $\varphi$ for which

$$
\begin{aligned}
& \lambda^{\prime}=\cos \omega(1+\rho \cos \varphi), \mu^{\prime}=\sin \omega(1+\rho \cos \varphi), \nu^{\prime}=\rho \sin \varphi, \\
& x=\psi_{1}(\omega), y=\psi_{2}(\omega), z=\psi_{3}(\omega)+\rho \cos \varphi, t=\psi_{4}+\rho \sin \varphi .
\end{aligned}
$$

By virtue of (4.17) and (4.16), $x, y, z, t$ are functions of $\omega, \rho, \varphi$ and, eventually, of $\lambda^{\prime}, \mu^{\prime}, \nu^{\prime}$. At this stage, however, $x, y, z, t$ are single-valued analytic functions of $\omega, \rho, \varphi$ but not of $\lambda^{\prime}, \mu^{\prime}, \nu^{\prime}$. However, if we agree that $\left.\rho \in\right] 0,1[$, then to a set of values $\lambda^{\prime}, \mu^{\prime}, \nu^{\prime}$ there corresponds a unique set of values of $\rho, \cos \omega, \sin \omega, \cos \varphi, \sin \varphi$, and hence a unique set of values of $x, y, z, t$. By virtue of this restriction, $x, y, z, t$ become eventually single-valued analytic functions of $\lambda^{\prime}, \mu^{\prime}, \nu^{\prime}$. To a point $\left(\lambda^{\prime}, \mu^{\prime}, \nu^{\prime}\right)$ satisfying the condition $\rho<1$ and hence lying within a certain torus, there corresponds one and only one point $(x, y, z, t)$.

Within this representation scheme, the curve $C$ is represented by the circle $(\rho=0)$

$$
\lambda^{\prime 2}+\mu^{\prime 2}=1, \nu^{\prime}=0 .
$$

The Poincaré choice for the surface $\Sigma$ is the torus

$$
\left.\rho=\rho_{0}, \rho_{0} \in\right] 0,1[
$$

that wraps the curve $C$. We can now decompose the integration over $\Sigma$ into two steps. First, we integrate over $\eta$, regarding $\xi$ as an arbitrary parameter. Hence we have

$$
z=\psi_{3}(\omega)+\rho_{0} \cos \varphi, t=\psi_{4}(\omega)+\rho_{0} \sin \varphi .
$$

If $\xi$ is viewed for a moment as a constant, $\omega$ is then also a constant; the same holds for $\rho_{0}, \varphi$ being the only independent variable, and we have

$$
\eta=\psi_{3}+i \psi_{4}+\rho_{0} e^{i \varphi},
$$

which shows that the point $\eta$ describes in the $\eta$-plane a circle of radius $\rho_{0}$ centred at the point $\psi_{3}+i \psi_{4}$. The integral

$$
I=\int F(\xi, \eta) d \eta
$$

is therefore equal to $2 i \pi$ times the residue of the function $F(\xi, \eta)$ (viewed as a function of $\eta$ only) with respect to the point $\psi_{3}+i \psi_{4}$. 
Along the singular curve $C$, the denominator $Q(\xi, \eta)$ in (4.13) vanishes:

$$
Q(\xi, \eta)=Q\left(\psi_{1}+i \psi_{2}, \psi_{3}+i \psi_{4}\right)=0 .
$$

In the final stage, one has to integrate with respect to $\xi$ by letting $\omega$ vary from 0 to $2 \pi$, i.e., upon following the whole singular curve $C$. We are therefore led to studying the integral

$$
J=\int 2 i \pi \operatorname{Res}\left(\frac{P(\xi, \eta)}{Q(\xi, \eta)}\right) d \xi
$$

along the curve $C, \eta$ being related to $\xi$ by the equation (4.23).

\subsection{The case of the scattering amplitude}

Since Poincaré restricted himself to rational functions $F=\frac{P}{Q}$, his condition $Q=0$ was an algebraic equation, unlike our case, where we propose to look for functions $P$ and $Q$ such that, by virtue of Eq. (1.2),

$$
2 i \pi \operatorname{Res} \frac{P(l, n(l))}{Q(l, n(l))}=\frac{1}{2 k}\left(e^{2 i \delta(\lambda, k)}-1\right) \frac{\lambda P_{\lambda-\frac{1}{2}}(-\cos \theta)}{\cos (\pi \lambda)},
$$

where on the right-hand side $\lambda$ is defined as in (2.9) but is viewed as a single complex variable.

\section{Concluding remarks}

The complex angular momentum formalism is still finding applications, not only to particle physics [15], but also to the scattering of scalar, electromagnetic and gravitational waves by a Schwarzschild black hole 16, 17. From the point of view of general formalism, Regge's method was remarkable because his use of Watson transform made it possible to elucidate analytic properties of the scattering amplitude. On the other hand, from the point of view of particle physics, if the variable $l$ is interpreted as the intrinsic angular momentum, a Regge singularity establishes a relation between spin and mass of the exchanged particle 6. In modern times, this picture is possibly superseded by the gauge theories of fundamental interactions, but one may instead appreciate the interest of our Sect. 3, where complex $\lambda$ is achieved by complexifying $n$ only. The complexification of $n$ may seem counterintuitive, but is perfectly consistent with the residue calculation in Eq. (1.3), and in our opinion it would be a lost opportunity to regard it as counterintuitive or a pure artifice devoid of physical interest.

In our paper we have then undertaken the task of preparing the ground for a derivation of the non-relativistic scattering amplitude from double integrals in the complex domain. Our contribution is so far at the level of ideas, but the picture outlined in this manuscript adds evidence in favour of new applications of complex analysis to quantum physics being in sight. 


\section{acknowledgements}

The author is grateful to the Dipartimento di Fisica "Ettore Pancini" for hospitality and support, and to Roland Rosenfelder and Gabriele Gionti for correspondence.

\section{References}

[1] T. Regge, Introduction to complex orbital momenta, Nuovo Cim. 14, 951976 (1959).

[2] A. Bottino, A.M. Longoni and T. Regge, Potential scattering for complex energy and angular momentum, Nuovo Cim. 23, 954-1004 (1962).

[3] V. de Alfaro and T. Regge, Potential Scattering (North-Holland, Amsterdam, 1965).

[4] G.N. Watson, The diffraction of electric waves by the earth, Proc. R. Soc. Lond. 95, 83-99 (1918).

[5] A. Sommerfeld, Partial Differential Equations in Physics (Academic Press, New York, 1949).

[6] A. Bottino, A retrospective look at Regge poles, arXiv:1807.02456 [physics.hist-ph].

[7] A. Chatterjee, Large-N expansions in quantum mechanics, atomic physics ands some $O(n)$ invariant systems, Phys. Rep. 186, 249-372 (1990).

[8] G. Esposito, Complex parameters in quantum mechanics, Found. Phys. Lett. 11, 535-547 (1998).

[9] E.T. Whittaker and G.N. Watson, Modern Analysis (Cambridge University Press, Cambridge, 1927).

[10] T. Daudé and F. Nicoleau, Local inverse scattering at a fixed energy for radial Schrödinger operators and localization of the Regge poles, Ann. H. Poincaré 17, 2849-2904 (2016).

[11] H. Poincaré, Sur les résidus des intégrales doubles, Acta Math. 9, 321-380 (1887).

[12] J. Leray, Le calcul différentiel et intégral sur une variété analytique complexe, Bull. Soc. Math. France 87, 81-180 (1959).

[13] L.A. Aizenberg and A.P. Yuzhakov, Integral Representations and Residues in Multidimensional Complex Analysis, Transl. Math. Mon. 58 (AMS, Providence, 1983).

[14] R. Caccioppoli, Theory of Functions of Several Complex Variables (Federico II University, Naples, 1947).

[15] V. Del Duca and L. Magnea, The long road from Regge poles to the LHC, arXiv:1812.05829 [hep-ph]. 
[16] A. Folacci and M.O. El Hadji, Regge pole description of scattering of scalar and electromagnetic waves by a Schwarzschild black hole, Phys. Rev. D 99, 104079 (2019).

[17] A. Folacci and M.O. El Hadji, Regge pole description of scattering of gravitational waves by a Schwarzschild black hole, Phys. Rev. D 100, 064009 (2019). 